\title{
The Mechanical and Thermal Design for the MICE Focusing Solenoid Magnet System*
}

\author{
S. Q. Yang, M. A. Green, G. Barr, U. Bravar, J. Cobb, W. Lau, R. S. Senanayake, A. E. White, and H. Witte
}

\begin{abstract}
The focusing solenoids for MICE surround energy absorbers that are used to reduce the transverse momentum of the muon beam that is being cooled within MICE. The focusing solenoids will have a warm-bore diameter of $470 \mathrm{~mm}$. Within this bore is a flask of liquid hydrogen or a room temperature beryllium absorber. The focusing solenoid consists of two coils wound with a copper matrix $\mathrm{Nb}-\mathrm{Ti}$ conductor originally designed for MRI magnets. The two coils have separate leads, so that they may be operated at the same polarity or at opposite polarity. The focusing magnet is designed so that it can be cooled with a pair of $1.5 \mathrm{~W}$ (at $4.2 \mathrm{~K}$ ) coolers. The MICE cooling channel has three focusing magnets with their absorbers. The three focusing magnets will be hooked together in series for a circuit stored-energy of about 9.0 MJ. Quench protection for the focusing magnets is discussed. This report presents the mechanical and thermal design parameters for this magnet, including the results of finite element calculations of mechanical forces and heat flow in the magnet cold mass.
\end{abstract}

Index Terms-Superconducting Solenoids, and Muon Cooling

\section{INTRODUCTION}

$\mathrm{T}$ he development of a muon collider or a neutrino factory requires that beams of low emittance muons be produced. A key to the production of low emittance muons is muon cooling. A demonstration of muon cooling is essential to the development of muon accelerators and storage rings [1], [2]. The international Muon Ionization Cooling Experiment (MICE) will be a demonstration of muon cooling in a configuration of superconducting magnets [3] that may be useful for a neutrino factory.

Ionization cooling of muons means that muons have their momentum reduced in both the longitudinal direction and the transverse direction by passing them through a low $\mathrm{Z}$ absorber. RF cavities are used to re-accelerate the muons to their original momentum. If the scattering in the absorbing medium is not too large, the reaccelerated muon beam will have a lower emittance than the muon beam that entered the absorbers. In order to reduce the multiple scattering of the muon beam in the absorber, the muon beam beta must be low in the absorber and the absorber must have a low $\mathrm{Z}$. The candidate absorbers include hydrogen (either as liquid or gas), lithium hydride, lithium metal or beryllium metal. The absorber in MICE is located within the absorber focus coil module where the beam has low beta (is well focused).

Manuscript received 5 October 2004. S. Q. Yang, M. A. Green (email MAGree@lbl.gov), G. Barr, U. Bravar, J. Cobb, W. Lau, R. S. Senanayake, A. E. White, and H. Witte are from the Oxford University Physics Department, Oxford OX1-3RH. *This work was supported by the Oxford University Physics Department and the Particle Physics and Astronomy Research Council of the United Kingdom.

\section{THE PROPOSED MICE EXPERIMENT}

The proposed MICE experiment will test cooling on a low intensity muon beam from the ISIS ring at the Rutherford Appleton Laboratory in the United Kingdom. The pions that decay into muons are produced by dipping a metal target into the ISIS proton beam. The muons will be collected and will be carried to an experimental hall containing the MICE.

The beam enters the experiment by passing through a foil that will scatter the muons to produce a beam with the desired emittance (beam scattering or beam transverse momentum). The muon emittance will be measured by four planes of scintillating fibers that are in a uniform (to about a percent) solenoidal magnetic field from 2.8 to $4 \mathrm{~T}$ [4]. Fig. 1 shows a schematic cross-section view of MICE that shows the detectors as well as the cooling channel.

Once the emittance of the muon beam entering the cooling section has been measured, the beam passes into the first absorber focus coil module (AFC module) The absorber cools the muon beam (reduces both the transverse and longitudinal momentum) by ionization cooling. The MICE absorbers may be liquid hydrogen, liquid helium, beryllium, or plastic. The preferred cooling absorber material is hydrogen, because it has the lowest multiple scattering of any of the absorber materials for a given energy absorption and beam beta.

The absorber is surrounded by a two-coil superconducting solenoid, which is the topic of this report. The magnet is designed to produce either a relatively uniform field or a cusp shaped field (a solenoidal quadruple field) that changes polarity as one goes along the axis. A key feature of the focusing magnet is the fact that the magnet is designed to operate as a two coil uniform field solenoid or as a gradient solenoid. When the focusing magnet operates in the gradient mode (with both coils at opposite polarity) the field is zero at the magnet center and the center of the absorber.

The muon beam longitudinal momentum is recovered by accelerating the beam with a four cell $201.25 \mathrm{MHz}$ RF cavity that are in the coupling coil 2.0 to $2.5 \mathrm{~T}$ magnetic field [5]. If the scattering within the absorber is low enough, the reaccelerated muon beam will be cooled. After the muon beam has been re-accelerated, it passes through a second absorber focus coil module for more beam cooling. The process of reacceleration is repeated then the beam passes through the final AFC module. At this point, the beam should be cooled enough, so that the emittance change can be clearly measured.

Once the beam has passed through the ionization cooling section, it enters the second detector section. The second detector section is identical to the first detector, except for the time of flight detectors at the end of the experiment. 


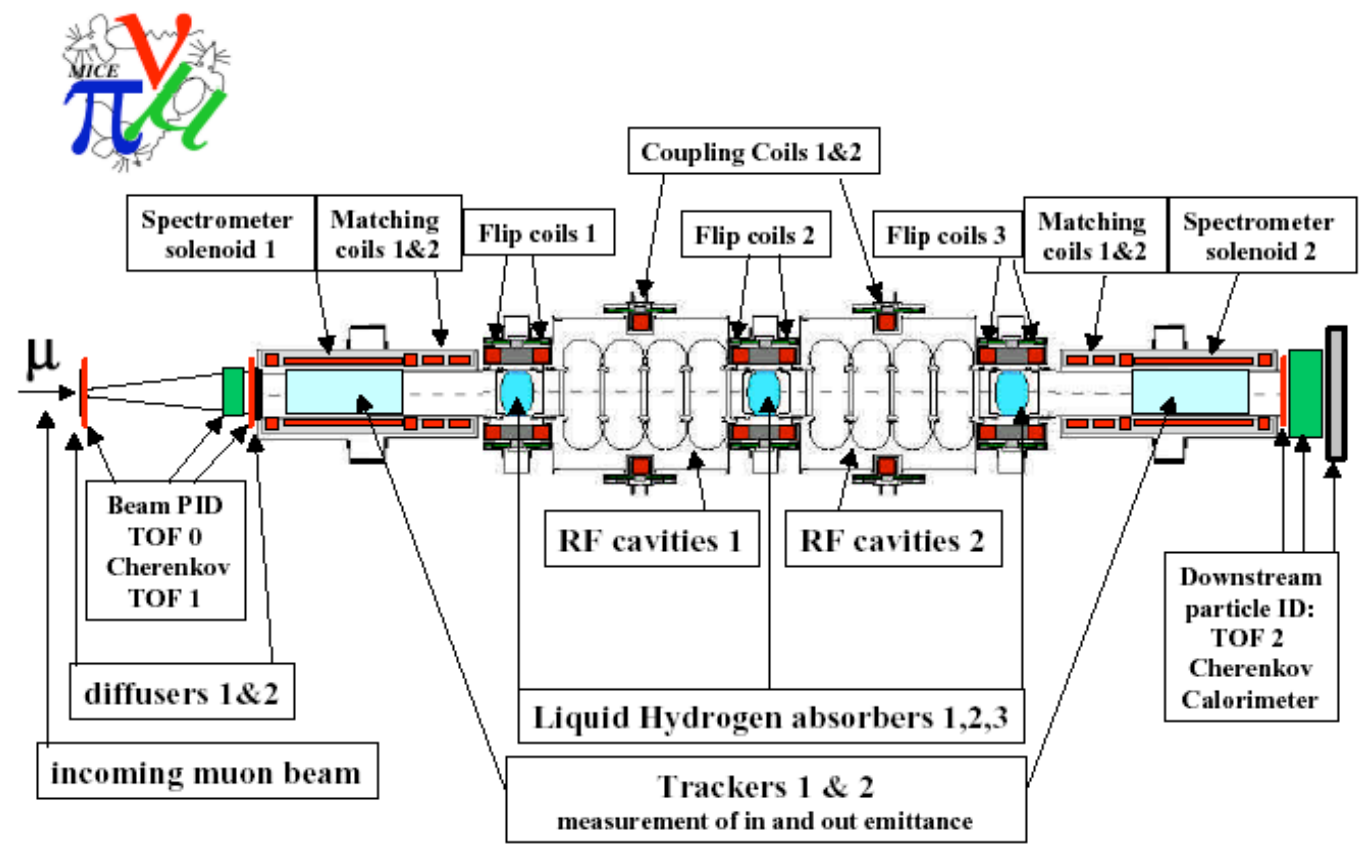

Fig. 1. A Schematic Representation of the MICE Experiment and its magnets shown in Cross-section. The absorber focus coil module (AFC module) consists of a liquid hydrogen absorber (or solid absorber) that is inside the focusing magnet (called the Flip coils).

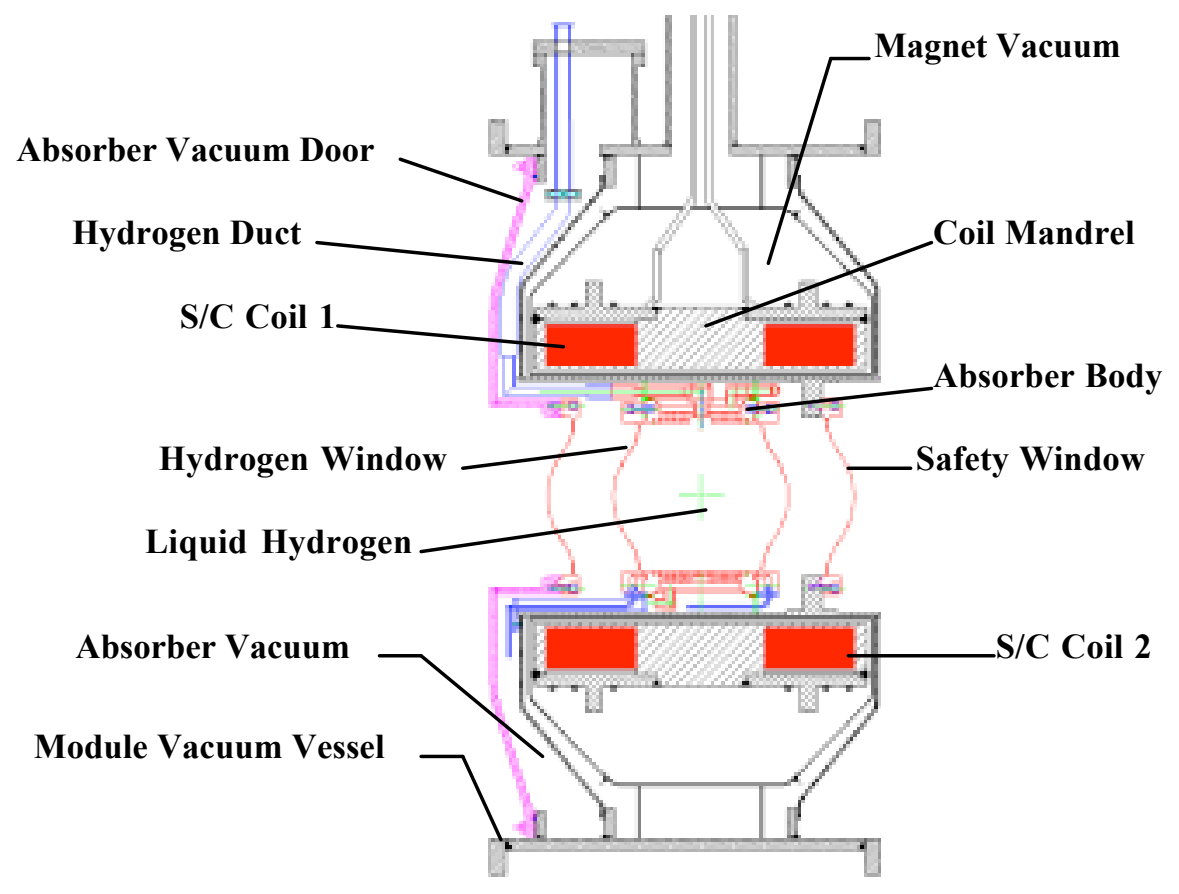

Fig. 2. A Schematic Representation of the MICE Absorber Focus Coil Module with a Liquid Hydrogen Absorber in the Magnet

\section{THE MICE ABSORBER FOCUS COIL MODULE}

The MICE absorber focus coil module (AFC module) consists of the focusing solenoid magnet, the muon absorber (either liquid or solid) within the warm bore of the magnet, the absorber plumbing, the magnet vacuum vessel, the absorber vacuum vessel, AFC module vacuum vessel, the liquid absorber cooler and a pair of $4.2 \mathrm{~K}$ coolers for keeping the magnet cold. A cross-section schematic representation of the AFC module with a liquid hydrogen absorber installed within the magnet bore is shown in Fig. 2. The AFC module is designed so that the magnet and the absorber can be tested separately away from where the MICE channel will be ultimately located. The AFC module design allows the AFC modules to be removed from the MICE channel, so that the absorbers can be changed from liquid absorbers to solid absorbers or from a solid absorber to a liquid absorber in a short time, while the focusing magnet remains at $4.2 \mathrm{~K}$

The cryostat vacuum for the magnet is completely separate from the absorber vacuum and the module vacuum. The module vacuum is common to the AFC module and the two 
modules next to the AFC module (either a RF module and a detector module or two RF modules depending on where the AFC is located within MICE). The vacuums are kept separate because one does not want frozen air gasses in contact with a vessel that contains liquid hydrogen. The MICE absorber is designed to run with liquid helium in the absorber as well as liquid hydrogen. As a result, the absorber vessel and its thin windows will be well insulated with MLI. The added MLI increase the effective thickness of the thin hydrogen windows about 17 percent.

The liquid hydrogen in the absorber is separated from the absorber vacuum by a thin window (about 120 micron thick) that is designed to have a room temperature burst pressure greater than $0.68 \mathrm{MPa}$. The diameter of the $20 \mathrm{~K}$ hydrogen thin window is $300 \mathrm{~mm}$. A room temperature $320-\mathrm{mm}$ diameter safety window (also 120 microns thick) with the same design burst pressure separates the absorber vacuum from the module vacuum.

The absorber and the focusing magnet will be cooled using three $4.2 \mathrm{~K}$ coolers. The absorber will be cooled using only one of these coolers. Cooling the absorber with a $4.2 \mathrm{~K}$ cooler permits MICE to be run with liquid helium in the absorber in place of the liquid hydrogen.

\section{The Focusing MAgnet Design}

The MICE focusing magnet has two $210 \mathrm{~mm}$ long coils wound on a 6061-T6-aluminum mandrel. The mandrel forms the 200-mm long spacer between the coils. The mandrel end flanges are $20 \mathrm{~mm}$ thick, so that the total length of the cold mass package (while warm) is $660 \mathrm{~mm}$. The inner bore radius of the cold mass is about $251 \mathrm{~mm}$ (at $300 \mathrm{~K}$ ). The 6061 aluminum mandrel and its aluminum covers carry the magnetic forces when the magnet operates in either the solenoid mode (the field is not flipped because the two coils are at the same polarity) or the gradient mode (the field is flipped along the magnetic axis because the two coils are at opposite polarity. This force can be as large as $3.53 \mathrm{MN}$ (360 metric tons) pushing the two coils apart. The focusing solenoid warm bore radius is $235 \mathrm{~mm}$. The length of the outside of the magnet cryostat vacuum vessel is $720 \mathrm{~mm}$.

The focusing magnet $\mathrm{Nb}-\mathrm{Ti}$ conductor is a conductor that is designed for use in MRI magnets. The bare dimensions of the conductor are $0.955 \mathrm{~mm}$ by $1.60 \mathrm{~mm}$, with rounded ends to prevent cracking of the Formvar insulation. The insulated dimensions of the conductor are $1.00 \mathrm{~mm}$ by $1.65 \mathrm{~mm}$. The conductor consists of four parts RRR $>75$ copper and one part $\mathrm{Nb}$-Ti. The superconductor is subdivided into 55 filaments, which are about 78 microns in diameter. The conductor twist pitch is about $12.7 \mathrm{~mm}$. The magnet has a layer glass fiber epoxy $0.1 \mathrm{~mm}$ thick between each layer of conductor. Thus the focusing magnet layer thickness shown in Table 1 is about $1.1 \mathrm{~mm}$.

Table 1 shows the basic parameters of the MICE focusing magnet. The magnet parameters are shown for both of the MICE magnet operating modes (where the magnetic field flips as one moves along the solenoid axis and where the field doesn't flip as one moves along the magnet axis). In both cases, the average momentum of the muons traveling along the MICE cooling channel is $240 \mathrm{MeV} / \mathrm{c}$ and the beam beta at the center of the absorbers is $420 \mathrm{~mm}$. Figure 3 shows the load lines for the flip and non-flip cases for the
MICE focusing magnet. The highest field and current points on the two lines correspond to the peak operating momentum of $240 \mathrm{MeV} / \mathrm{c}$ for MICE in both cases. The flip case (the open squares) operates with a much smaller temperature margin than the non-flip case (the closed circles).

TABLE 1.

THE BASIC PARAMETERS OF THE FOCUSING MAGNET IN THE NON -FLIP AND FLIP MODE

\begin{tabular}{lcc}
\multicolumn{1}{c}{ Parameter } & Non-flip & Flip \\
\hline Coil Separation (mm) & 200 & 200 \\
Coil Length (mm) & 210 & 210 \\
Coil Inner Radius (mm) & 263 & 263 \\
Coil Thickness (mm) & 84 & 84 \\
Number of Layers & 76 & 76 \\
No. Turns per Layer & 127 & 127 \\
Magnet J (A mm $\left.{ }^{-2}\right)^{*}$ & 71.96 & 138.2 \\
Magnet Current (A)* & 130.5 & 250.7 \\
Magnet Self Inductance (H) & 137.4 & 98.6 \\
Peak Induction in Coil (T)* & 5.04 & 7.67 \\
Magnet Stored Energy (MJ)* & 1.17 & 3.10 \\
4.2 K Temp. Margin (K)* & $\sim 2.0$ & $\sim 0.6$ \\
Inter-coil Z Force (MN)* $^{*}$ & 0.56 & 3.40 \\
\hline
\end{tabular}

* Design based on $\mathrm{p}=240 \mathrm{MeV} / \mathrm{c}$ and beta $=420 \mathrm{~mm}$

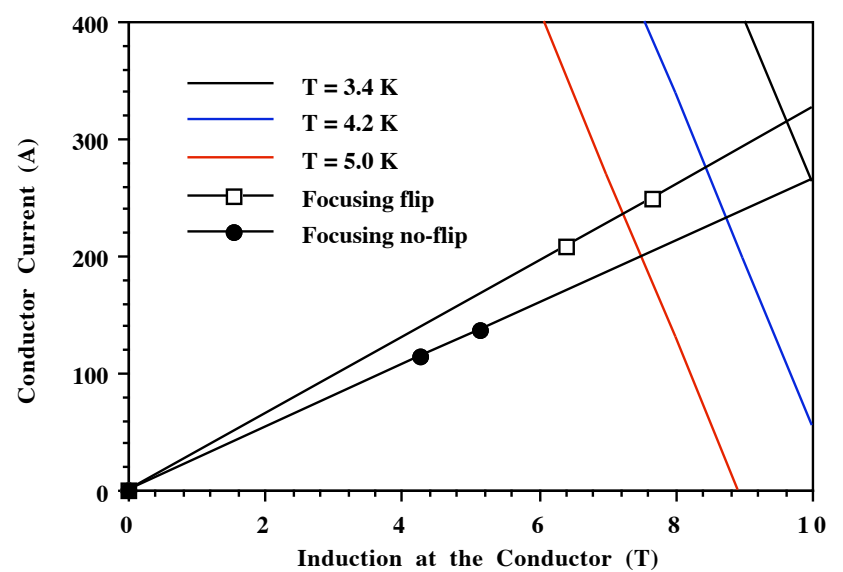

Fig. 3. The Magnet Load Lines and Conductor Current Versus the Magnetic Induction at Various Conductor Temperatures.

The non-flip case shown in Table 1 is not the worst-case non-flip mode that the MICE focusing magnet will see. There are some low beam beta modes that may drive the focusing magnet to higher currents in the non-flip mode than are shown in Table 1 or Fig. 3. The worst non-flip case has about the same temperature margin as the field-flip case shown in Table 1. In this case, the current in the coil is about $217 \mathrm{~A}$ and the magnet stored energy is about $3.2 \mathrm{MJ}$.

The focusing magnet stored energy is comparable to the Lab G solenoid tested at Fermilab [6], [7], which is the same size and uses the same conductor. The Lab G solenoid quenched safely with a hot spot temperatures less than $200 \mathrm{~K}$ even though the conductor ran at a higher current for the same stored energy. Like the Lab G solenoid, the MICE focusing 
magnet will be protected during a quench, by room temperature diodes across each coil and by quench back from the 6061 aluminum mandrel. Because there are warm diodes across each coil, the three focusing coils will be hooked up in series so that they all quench together.

The stress and deflection in the focusing magnet was analyzed using FEA methods. The largest stresses and deflections of the focusing magnet coils occur during the magnet cool down from $300 \mathrm{~K}$ to $4.2 \mathrm{~K}$ [8]. The aluminum mandrel and support structure is put into tension in both the longitudinal and the hoop directions during the cool down. The focusing coils are put into compression during the cool down. Powering the coil adds to the tensile stress in the aluminum. The largest von Mises stresses are found in the corners between the mandrel and the end plates and the cover structure and the end plates. These stresses are highly localized and will have little effect on the rest of magnet.

The focusing magnet cold mass support is a self-centering support system consisting of eight tension bands [9]. (The magnet center does not change as the magnet is cooled down.) The support system is designed to carry a sustained longitudinal force up to $500 \mathrm{kN}$ (50 tons) and transient forces up to $1000 \mathrm{kN}$ (100 tons).

\section{Cooling the Magnet with Small Coolers}

The MICE focusing magnets are designed to be cooled using a pair of small ( 1 to $1.5 \mathrm{~W}) 4.2 \mathrm{~K}$ coolers [10]. The second cooler is needed because the dominant heat load in the cooler first stage are four 300 A copper current leads. About half the heat leak into the $4.2 \mathrm{~K}$ region from the first stage temperature is down the four high temperature superconductor (HTS) leads that are connected to the room temperature current leads. The HTS leads are an enabling technology that permits magnets at $4.2 \mathrm{~K}$ to be continuously powered from a current source at room temperature.

Because the temperature margin in the focusing magnet is quite low at its maximum design current, it is important to minimize the temperature rise from the cooler second stage cold head and the hot spot in the magnet. (In the focusing magnet the hot spot in the magnet is very close to the high field point in the magnet winding.) First one must reduce the temperature rise within the magnet by applying the cooling evenly over the outside surface of the magnet [11]. Second, one must reduce the temperature drop from the point where the cooling is applied to the magnet surface and the cooler second stage cold head. Since the outside of the AFC module is some distance from the magnet cold mass, the use of a copper strap to carry the heat from the magnet cold mass to the cooler almost precluded. One can apply cooling to the outside surface of the coils by immersing them in liquid helium. The same liquid helium is an integral part of the gravity feed heat pipe that delivers the heat from the helium in the magnet to the cold head. Unlike conducting heat in a copper strap, the temperature drop along the heat pipe is independent of the distance between the cooler cold head and the surface of the magnet [10]. If the heat pipe is correctly designed, the temperature drop from the magnet hot spot to the $2^{\text {nd }}$ stage cold head of the coolers can be less than $0.2 \mathrm{~K}$.
It is possible to cool down the focusing magnet with the cooler, provided one uses a copper strap to carry the heat during the cool down. Since using the cooler to cool down, the magnet takes a long time (over 1 week), the focusing magnets are designed to be cooled down using liquid cryogens.

\section{CONCLUDING COMMENTS}

The focusing magnets for MICE can be built using commercial niobium titanium MRI conductors. The focusing magnets can be integrated with either liquid or solid absorbers in the magnet warm bore. The MICE focusing magnet are designed to be operated either as a split pair solenoid or as a gradient solenoid where the field flips polarity at the center of the magnet. Operation of the magnet in the gradient mode defines the design of the magnet.

The focusing magnet is designed so that it can be operated in a channel where the fields from other magnets that can interact with the focusing magnet. The stress levels in the magnet are reasonable even at the highest design currents.

The focusing magnet is designed to be cooled using a pair of two stage coolers that produce up to $1.5 \mathrm{~W}$ at $4.2 \mathrm{~K}$. The connection of the cooler to the magnet is designed to maximize the focusing magnet operating temperature margin.

\section{REFERENCES}

[1] N. Holtkamp and D. Finley Eds., "A Feasibility Study of a Neutrino Source Based on a Muon Storage Ring," FERMI-Pub-00/108E, (2000)

[2] R. B. Palmer, A. Sessler, A. Skrinsky, A. Tollestrup, et al, "Muon Colliders, " Brookhaven National Laboratory Report BNL-62740, January 1996

[3] M. A Green and J. M. Rey, "Superconducting Solenoids for an International Muon Cooling Experiment," IEEE Transactions on Applied Superconductivity 13, No. 2 p 1373 (2003)

[4] P. Fabricatore, S. Farinon, U. Bravar, and M. A. Green, "The Mechanical and Thermal Design for the MICE Detector Solenoid Magnet System," IEEE Transactions on Applied Superconductivity 15, (this volume), (2005)

[5] M. A. Green, S. Q. Yang, U. Bravar, et al, "The Mechanical and Thermal Design for the MICE Coupling Solenoid Magnet," IEEE Transactions on Applied Superconductivity 15, (this volume), (2005)

[6] M. A. Green, J. Y. Chen and S. T. Wang, "The Design, and Construction of a Gradient Solenoid for the High Power RF Cavity Experiment for the Muon Collider," Institute of Physics Conference Series 167, p 1199, (2000),

[7] M. A. Green, J. Y Chen, and S. T. Wang, "A Test of a Superconducting Solenoid for the MUCOOL RF Experiment," IEEE Transactions on Applied Superconductivity 11, p 2296, (2001)

[8] M. A, Green and S. Q. Yang, "The Coil and Support Structure Stress and Strain in the MICE Focusing and Coupling Magnets," an Oxford University Report, 30 August 2004, Contact S. Q. Yang of the Oxford University Physics Department for a copy.

[9] M. A. Green, R. S. Senanayake, "The Cold Mass Support System for the MICE Focusing and Coupling Magnets," an Oxford University Report, 23 August 2004. Contact S. Q. Yang of the Oxford University Physics Department (email: s.yang@physics.ox.ac.uk) for a copy.

[10] M. A. Green, "Cooling the MICE Magnets using Small Cryogenic Coolers," an Oxford University Report, 10 September 2004. Contact S. Q. Yang of the Oxford University Physics Department for a copy.

[11] M. A. Green, and S. Q. Yang, "Heat Transfer into and within the 4.4 K Region and the $40 \mathrm{~K}$ Shields of the MICE Focusing and Coupling Magnets," Oxford University Report with no formal number, 28 April 2004. Contact S. Q. Yang of the Oxford University Physics Department for a copy. 